\title{
LA DISTRIBUCIÓN DE LAS OCLUSIVAS ORALES EN LA TOPONIMIA PRERROMANA DE LA BÉTICA
}

\author{
José A. Correa
}

1.1. Cuando Plinio va a comenzar la enumeración de las ciudades de la Bética, anuncia que sólo va a nombrar ex his digna memoratu aut Latio sermone dictu facilia ( $N H$ 3,7). Esto bien puede interpretarse como una excusa de carácter tópico para justificar la selección que, más allá de las ciudades por todos conocidas, sin duda se ve obligado a hacer en una provincia en la que el desarrollo urbano es muy anterior a la presencia romana. No es creíble que a más de dos siglos de distancia de la conquista haya topónimos de "incómoda pronunciación" en una región profundamente romanizada. La ocupación del terreno llevada a cabo por los romanos debió obligarles a una rápida familiarización con los nombres de los lugares, por muy extraños que les sonaran, con adaptación inmediata a la fonética y morfología latinas: los topónimos son de uso obligado para todos y se imponen formas reconocibles. Esta es sin duda la razón de que tales topónimos, tal como nos los transmiten Plinio y otros autores o documentos, no suenen en modo alguno a algo extraño al latín.

El hecho de que casi todos los topónimos indígenas meridionales nos sean conocidos exclusivamente en su forma latinizada supone una fuerte limitación en su estudio formal, agravada por nuestro práctico desconocimiento de las lenguas habladas en el sur de la Península en el momento de la conquista (finales del s. III a. C.). Pero es seguro que no se trataba de una toponimia lingüísticamente uniforme sino formada dentro de diversas lenguas, algunas de las cuales pueden identificarse. ${ }^{1}$ Y esta situación se complica porque, a su vez, una toponimia de orígenes diversos, con posibilidad incluso de formaciones híbridas, puede haber sido tomada por los romanos total o mayoritariamente de una sola lengua, en la que habrá sufrido una inevitable uniformación previa.

Pero, a pesar de que las adaptaciones que ha podido sufrir un topónimo desde su nacimiento hasta su adopción obligada por los romanos han debido de ser múltiples, la uniformidad última que impone el latín no ha tenido por qué borrar totalmente algunas de las características más

\footnotetext{
${ }^{1}$ La cuestión ha sido tratada recientemente y con amplitud por Villar (2000).
} 
o menos generales que pueda tener esta toponimia. Sin duda es cierto que no pervivirá nada que no pueda ser encajado de alguna manera en la fonología y morfología del latín de ese momento, singularmente el inventario de fonemas, pero aquellas regularidades que no sean exigencia del latín es lícito atribuirlas a los topónimos en su etapa prelatina. Es en esta línea como van a ser enfocados algunos hechos que afectan a parte del consonantismo de estos topónimos.

1.2. Las observaciones que siguen se basan en un análisis de los dos centenares conocidos de topónimos prerromanos de la Bética, entendiendo por tales los que nombran lugares habitados. Parece un número suficiente para sacar conclusiones válidas, aunque de hecho son menos los que ofrecen información, pues no todos contienen consonantes oclusivas orales ${ }^{2} \mathrm{y}$, por otra parte, parece preferible prescindir de aquellos topónimos que podrían ser latinos, como los terminados en -ana, o cuya forma no está fijada con una cierta seguridad. ${ }^{3}$

Aunque está por hacer un estudio minucioso y detallado de la mayoría de estos topónimos, singularmente en lo que se refiere a la cantidad vocálica, a efectos de lo que aquí se pretende parece suficiente con las cautelas tomadas para la fijación de las respectivas formas. En efecto, son escasísimos los topónimos cuya forma prerromana es conocida directamente, en concreto, en leyendas monetales. Los demás, en su inmensa mayoría, son conocidos en forma ya latinizada y, de entre estos, no es alto el número de los que son conocidos directamente por inscripciones y monedas (con frecuencia se trata sólo del adjetivo toponímico) frente a los de transmisión exclusivamente literaria (y, por tanto, no tan fiable). ${ }^{4}$ En fin, algunos topónimos sólo se conocen helenizados, proceso que suele suponer una latinización previa, y hay topónimos que sólo se conocen en época tardía, por lo que han podido evolucionar ya en latín antes de presentarse ante nosotros.

Por ello en el catálogo de los topónimos de la Bética que aparece a continuación se ha dado preferencia al testimonio epigráfico o numismático (capitales) $)^{5}$ sobre el literario y al latino sobre el griego; asimismo se han señalado con un asterisco los topónimos no documentados como tales sino deducidos de su correspondiente adjetivo, que se añade. ${ }^{6}$

\footnotetext{
${ }^{2}$ En los hechos fonológicos que trato más abajo prescindo de las labiovelares como distintas de las velares, aunque en mi opinión las había en este conjunto toponímico: con seguridad en Munigua y probablemente también en Ategua y Osqua $(<* o s(s)$-gua?). Es cuestión que espero tratar en otro lugar. Asimismo no he considerado la presencia en algunos topónimos de $x$, pues podría representar una silbante especial en vez de una secuencia [ks].

${ }^{3}$ En cualquier caso los topónimos de los que he prescindido, aunque no sean claramente latinos, no serían objeción a las conclusiones que luego se sacarán. En el catálogo elaborado he seguido a Tovar (1974), actualizándolo fundamentalmente con CIL $\mathrm{II}^{2} / 5 \mathrm{y} \mathrm{II} / 7$.

${ }^{4}$ Para estos últimos algunas fuentes, como la Rauennatis anonymi cosmographia, son de escasa confianza.

${ }^{5}$ Utilizo capitales en cursiva para las escasas leyendas monetales púnicas. Prescindo de las libiofenicias por la inseguridad de su lectura y transcripción.

${ }^{6} \mathrm{Si}$ el adjetivo está documentado sólo en inscripciones perdidas y, por tanto, de lectura no comprobable, va asimismo precedido de asterisco.
} 
1.3. Catálogo de topónimos prerromanos de la Bética examinados:

'BDRT - ABDERA, ABRA, ACINIPO, Agla ${ }^{7}$ Ali yova, AIPORA, ${ }^{8}$ *Aiungi (AIVNGITANVS), *Andura (ANDVRENSIS), Anticaria (ANTIK(ariensis)), *Aratispi (ARATISPITANVS), 'Aркı入акís, Arialdunum, Artigi, ${ }^{9}$ Arucci, Arunda, 'SDN - ASIDO, Aspauia, Astapa, ASTIGI, Ategua, *Aurgi (AVRGITANVS), Axati, *Baedro (BAEDRONENSIS), $B Y L ' N N$ - BAILO, B(a)esaro, Baesippo, *Baesucci (BAESVCCITANVS), Baîtıs, BAICIP(O), Baldo/Bardo, Barbesula, Basilippo, *Batora (BATORENSIS), *Baxo (BAXONENSIS), Belippo, BORA, Brona,${ }^{10}$ BRVTO-

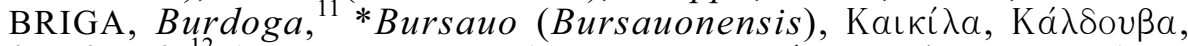
* Calecula ${ }^{12}$ (CALECVlensis), CAllet, Kávaka, *Canania (CANAN(iensis)), *Cantigi (CANTIGIT[anus]), Cappa, CARBVLA, CARI(S)SA, CARMO, Carruca, CARTEIA, Cartima, Carula, Cauiclum, CAVRA, *Cedrippo (*CEDRIPPONENSIS), Celti (CELTITAN(um)), ${ }_{15}^{13}$ CERI, ${ }^{14}$ CILPE, Cisimbrium, CORDVBA, CVNBARIA - CONOBARIA, ${ }^{15}$

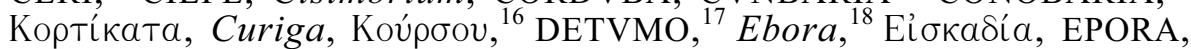

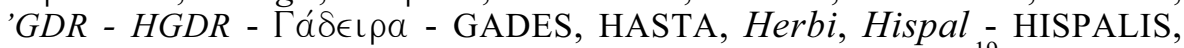
*Igabrum (IGABRENSIS), ILIBERI (ILIBERRITA(num)), ${ }^{19}$ Ilipa (ILIPENSE), ${ }^{20}$ ILIPLA, Ilipula, ${ }^{21}$ ILVTVRGI ${ }^{22}$ - Iliturgi, * Iliturgicola (ILITVRGICOLENSIS), *Ilurco (ILVRCON(ense)), ${ }^{23} *$ Ipagrum (*IPA-

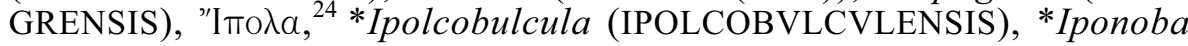

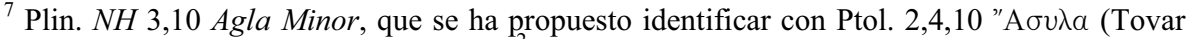
(1974), p. 181). Es posible que en $C I L \mathrm{II}^{2} / 5,280$ (inscr. perdida) Agul(ensis?) esté documentado el correspondiente adjetivo.

${ }^{8}$ De identificación discutida (TIR, s. u. Aipora/Ebora).

${ }^{9}$ Hay dos ciudades del mismo nombre, una al sur del Guadalquivir y otra identificable posiblemente con Castuera (Badajoz) (CIL $\mathrm{II}^{2} / 7$, p. 216).

${ }^{10}$ Transmitido, al parecer, sólo por Plinio $N H 3,15$, plantea problemas su forma exacta.

${ }^{11}$ Testimoniado sólo por Rauen. 317,7.

${ }^{12}$ En Plin. NH 3,12 ningún manuscrito da la forma exacta; con variantes en Ptol. 2,4,9 y 10

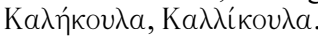

${ }^{13}$ Leyenda monetal. CELTE en un plomo monetiforme según Casariego (1987).

${ }^{14}$ Sólo testimonio monetal; también CER+, que a veces parece CERI según Villaronga (1994), p. 387, y que Vives (1924, IV), p. 78, leyó CERT.

${ }^{15}$ CVNBARIA es leyenda monetal. Se suele identificar con Plin. NH 3,11 Colobana.

${ }^{16}$ TIR, s. u. Curiga, la identifica con esta; Tovar (1974), p.179, es de la opinión contraria.

${ }^{17}$ No concuerdan los hallazgos monetales (entre Jerez de la Frontera y Arcos) y la localización dada por Plin. $N H$ 3,10 junto al Guadalquivir (hacia Palma del Río, Córdoba). Se suele identificar con Ptol. 2,4,9 $\Delta \in T$ Toû $\delta a$.

${ }^{18}$ Hay dos ciudades homónimas: Mela 3,4 castellum Ebora, Plin. NH 3,10 Ebora quae Cerialis. Se discute la adscripción de la moneda con leyenda EBOR(a) (TIR, s. uu. Aipora/Ebora y Ebora).

${ }^{19}$ Leyendas monetales.

${ }^{20}$ Leyenda monetal.

${ }^{21}$ Plin. NH 3,12 Ilipula Minor; 3,10 Ilipula quae Laus (corrección de Ilypula). Por otra parte es problemática la interpretación de las monedas con leyenda ILIPV // HALOS.

${ }^{22}$ Leyenda monetal; también ILDITVRGENSE.

${ }^{23}$ Leyenda monetal.

${ }^{24}$ Filóstrato, Vita Apol. 5,9.
} 
(IPONOBENSIS), IPORA, *Iporca (*IPORCENSIS), Ipra, *Ipsca (IPSCENSIS), IPTVCI, IRIPPO, Isturgi, ITVCI, ${ }^{25}$ *Iulipa (IVLIPENSIS), $\Lambda$ aKBís, Lacimurga, LACIPO, Lacunis, ${ }^{26}$ L(a)epia, LASCVT, ${ }^{27}$ LASTIGI,

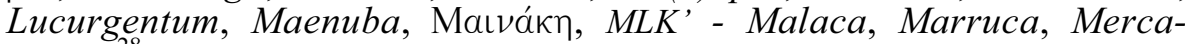
blum, ${ }^{28}$ Mirobriga, Munda, *Munigua (MVNIGVENSIS), Murgi, NABRISSA, Nertobriga, *Nescania (NESCANIENSIS), OBA, ibolka OBVLCO, Obulcula, OQVR ${ }^{29} *$ Oducia (*ODVCIENSIS), *Olba (OLBENSIS), OLONT(igi), ${ }^{30} *$ Onigi (ONIGITANVS), Onoba, ONVBA, ORIPPO (ORIPENSE), ${ }^{31}$ Osca, Oscua (OSQ(uensis)), O(S)SET, Ossigi, Ostippo, OSTVR, Пa $\sigma o v \lambda \lambda a,{ }^{*}$ Sabetum (*SABETANVS), Sabora, SACILI, Saepo, Saguntia, Salduba, SALPESA (SALPENSANVS), Saudo, Segida ${ }^{33}$ Segouia, Serippo, Serpa (SIRPENS(se)), ${ }^{34}$ Singili Barba ${ }^{35}$ SISAPO, SISIPO, Soricaria, *Sosontigi (SOSONTIGITANVS), ${ }^{36}$ Spalis, Sucaelo, Tema, ${ }^{37}$

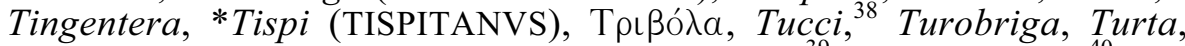
Vcia, Vcubi, VENTIPO, VESCI, VGIA, Vnditanum, ${ }^{39}$ Vrgao, Vrgapa. ${ }^{40}$

2.1. En inicial de topónimo para cada punto de articulación se conoce una sola consonante: $/ \mathrm{b} /, / \mathrm{t} /, / \mathrm{k} /$. Aunque hay excepciones, estas tienen claramente el carácter de tales en el conjunto de la toponimia y, si aparecen, es porque el latín no conocía una limitación de ese género en comienzo de palabra.

\footnotetext{
${ }^{25}$ Hay dos ciudades homónimas, una emite moneda y otra es citada por Plin. NH 3,12.

${ }^{26}$ Sólo conocido por Rauen. 314,15, se piensa que puede ser una transformación o deformación de Laci(ni)murga.

${ }^{27}$ Lascuta en Plin. 3,15 es corrección de lascula, liscula. El adjetivo es LASCVTANVS (CIL II 5041).

${ }^{28}$ It. Ant. 408,2 Mercablo (var. Mergablo).

${ }^{29}$ Leyenda monetal. Aunque se conoce epigráficamente el adjetivo OCVRITANVS, no se puede asegurar que sea OCVR(i) (cf. OSTVR).

${ }^{30}$ Leyenda monetal: también OLVNT(igi). Plin. NH 3,12 Alontigi, Mela 3,5 Olintigi.

${ }^{31}$ Leyendas monetales.

${ }^{32}$ Así Tovar (1974), p. 61: en Plinio NH aparecen 3,14 S(a)epone (abl. entendido como nominativo), 3,15 Vsaepo (mala interpretación de V(ictrix) Saepo). Parece, sin embargo, que eran dos ciudades distintas.

${ }^{33}$ Hay dos ciudades homónimas. Se conoce además un plomo monetiforme con la leyenda SEGEIDA.

${ }^{34}$ Leyenda monetal.

${ }^{35}$ Plin. NH 3,10 Singili (pero, como sigue Ategua, podría ser Singilia), It. Ant. 412,1 Barba. En las inscripciones están documentados tanto Singiliensis como Barbensis, casi exclusivamente abreviados y a veces juntos $\left(C I L \mathrm{II}^{2} / 5,772\right.$ ss. $)$.

${ }^{36}$ Plin. NH 3,14 Sosintigi.

${ }^{37}$ Documentado sólo en Rauen. 317,12. TIR, s. u. Laelia, la identifica, al parecer, con esta.

${ }^{38}$ Hay, al menos, dos ciudades homónimas: Plin. NH 3,12 Tucci quae cognominatur Augusta Gemella; id. 3,10 Tucci Vetus. Además It. Ant. 432,2 Tucci (entre Ilipla e Italica).

${ }^{39} \mathrm{Da}$ la impresión de ser en su origen un adjetivo sustantivado correspondiente a un topónimo relacionado etimológicamente con Arunda y Munda.

${ }^{40}$ Documentado sólo en Rauen. 316,6.
} 
Frente a casi una veintena de topónimos iniciados por /b/ sólo co-

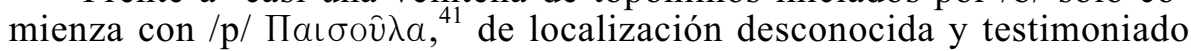
únicamente en Ptolomeo. Su segmento inicial parece igual al de los latinizados Baes-ippo y Baes-ucci, sólo que con ensordecimiento de la labial, lo que invita a un análisis [pais-ula]. ${ }^{42}$

Para las dentales junto a siete topónimos iniciados por /t/ sólo comienza con /d/ Detumo. Y frente a casi una treintena de topónimos ini-

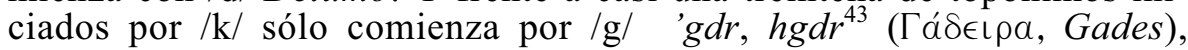
cuyo origen fenicio es indudable.

2.2. Respecto al contorno vocálico, en cualquier posición, sólo hay que destacar que /p/ no va precedida de /o/ ni /u/ y /t/ de /o/, lo que en conjunto carece de relevancia.

Respecto a las consonantes que pueden preceder a las oclusivas orales y prescindiendo precisamente de estas mismas, que sólo aisladamente admiten tal posición (Abdera, Iptuci, Ipsca), se pueden señalar algunas ausencias: a) /s/ sólo precede a las oclusivas sordas, lo que es un condicionamiento latino bien conocido; b) una nasal, cuyo punto de articulación en tal posición está condicionado en latín, no precede a $/ \mathrm{p} / \mathrm{y}$ $/ \mathrm{k} /$; c) $/ \mathrm{l} /$ no precede a $/ \mathrm{g} /$.

Las combinaciones documentadas con vibrante o lateral son (entre paréntesis los hápax): /br/, /bl/ (Mercablum);/pr/ (Ipra), /pl/ (Ilipla);

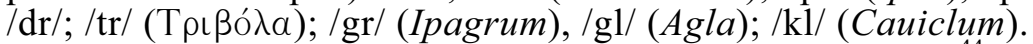

Sólo conocen geminación $/ \mathrm{p} /(\text { como variante de -ipo })^{44} \mathrm{y} / \mathrm{k} /($ como variante de $(t) u c i)$.

2.3. En final absoluto de topónimo sólo aparece, muy reducidamente, /t/, que es la única oclusiva oral abundante en esta posición en latín, si bien casi sólo en formas verbales personales.

3.1. Parte de estos hechos por sí mismos no son indicativos de una distribución fonólogica singular. En concreto, tratándose de secuencias de fonemas $(\$ 2.2)$ el número de posibles combinaciones es lo suficientemente alto como para que sea normal que haya combinaciones no documentadas. Así no estaría justificado proponer a título de hipótesis que la ausencia de nasal ante $/ \mathrm{p} / \mathrm{y} / \mathrm{k} /$ se debería a una sonorización de estas por influjo de aquellas, porque la secuencia/nt/ está bien documentada y

${ }^{41}$ No hay datos suficientes para suponer la existencia de un topónimo Polco $\left(C I L \mathrm{II}^{2} / 5,218\right.$, y p. 55).

${ }^{42}$ Villar (2000), p. 332, considera * pais- distinto de *bai-, si bien ambos tendrían etimología indoeuropea.

${ }^{43}$ En las leyendas monetales al topónimo propiamente dicho le precede el artículo.

${ }^{44}$ También en Cappa, de análisis contradictorio en Villar (2000): C-ap-pa (p. 176); sufijo -pa geminado, es decir, Ca-ppa (p. 94, n. 32). En mi opinión es preferible un análisis Cap-pa, pues Balsilippa, citado por Villar como testimonio complementario de la geminación del sufijo - pa, es sólo lectura de uno de los manuscritos de Rauen. 316,13 (los otros dos dan Balsilipa), que contradice la de It. Ant. 410,4 Basilippo, mucho más fiable.

${ }^{45} \mathrm{Me}$ parece poco probable, por la singularidad de su formación en la zona, que $C I L \mathrm{II}^{2} / 5$, 351 Siccaenas sea derivado de un indígena Siccaenum / Siccaena. 
ningún caso conocido de $/ \mathrm{mb} / \mathrm{y} / \mathrm{ng} /$ invita a considerarlo como producto de una sonorización. Tampoco cabe sacar consecuencias de las combinaciones con vibrante o lateral, aunque sólo /br/ y / dr/ superan la unidad; pero el número de testimonios es bajo.

3.2. La geminación, en cambio, sí aporta datos de interés. Desde el punto de vista de la fonología latina, y por tanto también de la escritura, sólo cabe que aparezca en posición intervocálica ${ }^{46}$ y casi siempre lo hace en juntura de morfema. Pero en estos topónimos debe corresponder a una particular articulación de las oclusivas sordas (de ahí las oscilaciones gráficas), presente sólo en interior de elemento toponímico (-ipo, ${ }^{47}$ (t) $u c i)$. En consecuencia ni se geminan las sonoras (-oba, -igi), ni la oclusiva sorda inicial de un elemento toponímico aunque en el compuesto resultante esté en posición intervocálica (/t/ en -tuci). Por otra parte, como no se conoce ningún elemento repetido en una serie de topónimos que tenga en su interior / $/$ / intervocálica, no es esperable que haya datos de la geminación de esta oclusiva.

3.3. El hecho sin duda más sorprendente es el que afecta a la limitación, ya señalada, que hay en inicial de topónimo (sólo $/ \mathrm{b} /, / \mathrm{t} / \mathrm{y} / \mathrm{k} /)^{48} \mathrm{y}$ obliga a preguntarse si en el momento en que se inicia la colonización romana no habría ya generalizada en la Bética una lengua que habría actuado como un filtro, dando una uniformidad fonológica a lo que sin duda era de origen variado, y ello en coexistencia con otras lenguas que se seguirían hablando en lugares y zonas limitadas.

Es cierto que el ibérico, que probablemente se hablaba en la zona oriental de la Bética, carece de /p/. Lo mismo se podría decir de la lengua celta que se puede suponer hablada en los lugares cuyo nombre es claramente celta. ${ }^{49}$ Pero respecto al turdetano o a cualquier otra lengua que se hablara simultáneamente en la Bética no se puede hacer ninguna afirmación. ${ }^{50} \mathrm{Y}$ en cualquier caso / $\mathrm{p} /$ era conocido como fonema, puesto que aparece abundantemente en interior de palabra y en plena coexistencia con $/ \mathrm{b} /$. Con más contundencia aún se puede argumentar a propósito de las oclusivas dentales y velares, ya que en ibérico tanto sordas como sonoras son conocidas en inicial de palabra y no hay razón para que no sucediera lo mismo en una lengua celta. ${ }^{51}$

\footnotetext{
${ }^{46}$ Ciertamente en época arcaica hay aisladamente geminadas en final absoluto, si bien nunca escritas.

${ }^{47}$ No hay datos seguros de geminación cuando se trata de ip-.

${ }^{48}$ Aunque no es uno de los fonemas tratados aquí, no carece de interés señalar que en la toponimia indígena tampoco / $\mathrm{r} /$ está documentado con seguridad en posición inicial, limitación que es típica del ibérico y, al parecer, de la lengua de las inscripciones en escritura "tartesia".

${ }^{49}$ Cabe ciertamente la posibilidad de que tal lengua, que no es inevitable identificar con el celtíbero, hubiera adquirido secundariamente el fonema $/ \mathrm{p} /$.

${ }^{50}$ Sería arriesgado ligar esto con el hecho de que la escritura paleohispánica, que se supone que se crea en esta zona a partir de la fenicia, no distingue entre oclusivas orales sordas y sonoras, pues se trata de realidades distanciadas probablemente por más de cuatro siglos.

${ }^{51}$ No hay tampoco limitación conocida para el uso de las oclusivas orales en el púnico.
} 
Por otra parte, al analizar los topónimos mediante comparación entre ellos, se pueden obtener algunos elementos toponímicos (naturalmente en posición no inicial) que comienzan por consonantes distintas de las señaladas: el más seguro es - $p a$. Pues bien, no se puede decir que la forma teóricamente admisible en posición inicial /ba/ sea aislable como elemento toponímico en esa posición. En consecuencia, no cabe tampoco pensar sin más en una neutralización de la correlación de sonoridad en función de la posición en la palabra, sino en que unidades como /pa/ funcionan como sufijos y no como miembros (intercambiables) de compuesto.

En fin, este hecho fonológico, que no parece que deba atribuirse a una casualidad, ${ }^{52}$ debe ser tenido en cuenta a la hora de rastrear la situación lingüística de la Bética en los comienzos de la latinización y en el análisis de la toponimia correspondiente.

\section{BIBLIOGRAFÍA}

Casariego, A., Cores, G., Pliego, F.(1987): Catálogo de plomos monetiformes de la Hispania antigua, Madrid.

Tabula Imperii Romani. Hoja-29: Lisboa (TIR), CSIC, Madrid, 1995.

TOVAR, A. (1974): Iberische Landeskunde. I, Baetica, ed. Valentin Koerner, Baden-Baden.

VILLAR, F. (2000): Indoeuropeos y no indoeuropeos en la Hispania prerromana. Las poblaciones y las lenguas prerromanas de Andalucía, Cataluña y Aragón según la información que nos proporciona la toponimia, Universidad, Salamanca.

Villaronga, L. (1994): Corpus nummum Hispaniae ante Augusti aetatem, ed. José A. Herrero, Madrid.

VIVES Y ESCUDERO, A. (1924): La moneda hispánica. I-IV, Ed. Reus, Madrid.

José Antonio Correa Rodríguez

Universidad de Sevilla

e-mail: jcorrea@siff.us.es

\footnotetext{
$\overline{52}$ La realidad es que una situación similar se advierte en los topónimos bastetanos y oretanos y en los lusitanos limítrofes, si bien el conjunto de esta toponimia es numéricamente inferior al de la Bética.
} 